\title{
CP.01. Apolipoprotein E polymorphisms in Brazilian children with heavy burden of diarrhea and consequences for later development.
}

\author{
ORIÁ, R.B. ${ }^{1}$, LIMA, A.A.Mํ․ GUERRANT, RL² \\ ${ }^{1}$ Faculty of Medicine, Federal University of Ceara \\ ${ }^{2}$ Center for Global Health, University of Virginia
}

Our group and others have reported a series of studies showing that heavy burdens of diarrheal diseases in the formative first two years of life in children in urban shantytowns have profound consequences of impaired physical and cognitive development lasting into later childhood and schooling. Environmental enteropathy (with recurrent episodes of enteric infections/malnutrition) afflicts young children living in impoverished areas of the developing world and causing a syndrome of chronic intestinal malabsorption, that ultimately leads to growth stunting and cognitive decline in times of important developmental milestones, therefore jeopardizing one's full genetic potential. Based on these previous studies showing that apolipoprotein E4 (APOE4) is relatively common in favela children, we review recent data suggesting a protective role for the APOE4 allele in the cognitive and physical development of children with heavy burdens of diarrhea in early childhood. APOE4 is relatively common in shanty town children living in Brazil (13.4\%) and suggest that APOE4 has a protective role in cognitive development as well as weight-for-height in children with heavy burdens of diarrhea in early childhood (64/123; 52\%), despite being a marker for cognitive decline with Alzheimer's and cardiovascular diseases later in life. APOE2 frequency was higher among children with heaviest diarrhea burdens during the first 2 years of life, as detected by PCR using the restriction fragment length polymorphism method, raising the possibility that ApoEcholesterol balance might be critical for growth and cognitive development under the stress of heavy diarrhea burdens and when an enriched fat diet is insufficient. Despite being a marker for cognitive decline with Alzheimer's and cardiovascular diseases later in life, APOE4 appears to be important for cognitive development under the stress of heavy diarrhea. In collaboration with Dr. Michael Vitek and Patrick Sullivan (Duke University, North Carolina), we have assessed different apoE genetic backgrounds, including apoE deficient and apoE target replacement mice carrying either E4 or E3 human genes, and found that cryptosporidial infections caused less growth decrement, less histologic damage and lower cryptosoridial infection burdens (by qPCR) and increased ileal CAT-1 expression in the ApoE4 targeted transgenic mice, all suggesting potential mechanisms by which ApoE4 may be protective in young children exposed to heavy diarrhea burdens and enteric pathogens in formative early life and perhaps even provide a basis for this allele in human evolution. The reviewed findings provide a potential explanation for the survival advantage in evolution of the thrifty APOE4 allele and raise questions about its implications for human development under lifestyle changes and environmental challenges.

Funding: USA National Institutes of Health ICIDR (\#5-UOI AI 26512-14), ABC (\#5D43 TW01136-04), TMRC (\#5 P50 AI30369-09), and FIC (\#TW006713-01) grants, and the Brazilian CNPq support.

ORIÁ, R.B.; LIMA, A.A.M.; GUERRANT, R.L. 2013. Apolipoprotein E polymorphisms in Brazilian children with heavy burden of diarrhea and consequences for later development, p.5. In: Oriá, Reinaldo Barreto; Andrade, Geanne Matos de; Bruin, Veralice Meireles S. de. I International Symposium in Neuroscience Meeting [Blucher Neuroscience Proceedings n.1 v.1]. São Paulo: Blucher, 2014, http://dx.doi.org/10.5151/isnm-sine1 